\title{
Intra-acciones en el aprender de docentes de infantil, primaria y secundaria
}

\section{Intra-actions in the learning of infant, primary and secondary teachers}

\author{
Juana María Sancho-GiL*1 \\ jmsancho@ub.edu \\ José Miguel Correa-Gorospe** \\ jm.correagorospe@ehu.eus \\ * Universidad de Barcelona, España \\ ** Universidad del País Vasco, España
}

\section{Resumen:}

En este artículo, desde la perspectiva de los nuevos materialismos y empirismos, nos acercamos a algunas formas, modos, momentos y lugares de aprender de docentes de infantil, primaria y secundaria. Lo hacemos, no solo centrándonos en las interacciones con sus contextos, sino desde la noción de las "intra-acciones" para ir más allá de la metafísica del individualismo que subyace a las interpretaciones convencionales de las "interacciones". Esto nos permite sobrepasar la separación que tiende a realizar la investigación lógicopositivista entre las personas y los objetos del mundo que les rodea, en este caso los contextos de aprendizaje de los docentes, al considerar que están interconectados antes de que la mirada del investigador los separe. Las cartografías de aprendizaje y las narraciones realizadas por los docentes que participaron en la investigación y las conversaciones mantenidas con ellos, nos han permitido identificar las intra-acciones de los espacios, tiempos, condiciones, ex-

\begin{abstract}
:
This article aimed to approach some forms, modes, moments and places of learning of infant, primary and secondary teachers from the perspective of new materialism and empiricism. We did this, not only by focusing on interactions with their contexts, but also from the notion of "intra-actions" since our purpose was to go beyond the metaphysics of individualism which underlies conventional interpretations of "interactions". This allowed us to overcome the separation that logical-positivist research tends to make between people and objects. This article, therefore, considered that in teacher learning contexts teachers and objects are interconnected before the researcher's gaze separated them. The learning cartographies and narrations provided by the teachers who participated in the research and the conversations held with them allowed us to identify in their learning processes the intra-actions of spaces, times, conditions, expectations, resources, artefacts, etc. This
\end{abstract}

1 Dirección para correspondencia (correspondence address):

Juana María Sancho-Gil. Universidad de Barcelona. Departamento de Didáctica y Organización educativa. Facultad de Educación. P. Vall d'Hebron, 171. 08028 Barcelona (España). 
pectativas, recursos, artefactos, etc., en sus procesos del aprender. Esto nos ha permitido vislumbrar la constitución mutua de organismos enredados y los difuminados límites entre los cuerpos y objetos, considerándolos fenómenos materiales discursivos. Desde una mirada post-cualitativa, situamos este análisis desde la teoría y lo que ésta permite pensar para que, más que 'obtener resultados', podamos contribuir a comprender el aprendizaje de los docentes y sus implicaciones para la práctica y la formación inicial y permanente. El artículo finaliza planteando la implicaciones y desafíos de este estudio en la forma de entender el aprender y en la formación del profesorado.

\section{Palabras clave:}

Investigación post-cualitativa; nuevos materialismos; nuevos empirismos; experiencias de aprendizaje; contextos de aprendizaje, cartografías. enabled us to glimpse the mutual constitution of entangled organisms and the blurred boundaries between bodies and objects, considering them as discursive material phenomena. From a post-qualitative perspective, we situated this analysis from what the theory allowed us to think so that, rather than 'obtaining results', we contributed to understanding teacher learning and its implications for practice and initial and ongoing training. The article ended by posing implications and challenges regarding the usual understanding of both learning and pre-service and in-service teachers' professional development.

\section{Key words:}

Post-qualitative research; new materialisms; new empiricisms; learning experiences; learning contexts; cartographies.

\section{Résumé:}

Dans cet article, nous abordons, du point de vue des nouveaux matérialismes et empirismes, certaines formes, modes, moments et lieux d'apprentissage à partir de la cartographie d'apprentissage d'enseignants de maternelle, du primaire et du secondaire. Nous le faisons en nous concentrant sur les interactions avec leurs contextes, mais aussi à partir de la notion d'»intra-actions», pour nous affranchir de la métaphysique de l'individualisme qui sous-tend les interprétations classiques des «interactions». Cela nous permet de dépasser la séparation que la recherche logico-positiviste tend à établir entre les personnes et les objets du monde environnant, en l'occurrence les contextes d'apprentissage des enseignants, considérant qu'ils sont interconnectés avant que le regard du chercheur ne les distingue. Les cartographies d'apprentissage et les récits des enseignants qui ont participé à la recherche et les conversations tenues avec eux nous ont permis d'identifier les intra-actions des espaces, des temps, des conditions, des attentes, des ressources, des artefacts, etc., dans les processus d'apprentissage d'enseignants. Cela nous a permis d'entrevoir la construction mutuelle d'organismes emmêlés et les brouillages de frontières entre corps et objets considérés comme des phénomènes matériels discursifs. D'un point de vue post-qualitatif, nous rattachons cette analyse à la théorie et à ce qu'elle nous permet de penser de manière à ce que, plutôt que 'd'obtenir des résultats', nous puissions contribuer à comprendre l'apprentissage des enseignants et ses implications pour la pratique et la formation initiale et continue. L'article se termine en présentant les implications et les défis de cette étude pour la compréhension de l'apprentissage et la formation des enseignants.

\section{Mots clés:}

Recherche post-qualitative; nouveaux matérialismes; nouveaux empirismes; expériences d'apprentissage; contextes d'apprentissage, cartographies.

Fecha de recepción: 22-11-2018

Fecha de aceptación: 14-5-2019 


\section{Introducción}

"El verdadero descubrimiento no consiste en encontrar nuevos territorios, sino en verlos con nuevos ojos". Marcel Proust

Este artículo toma como punto de partida la argumentación de Phillips (2014) de que el aprendizaje es un proceso profundamente contextualizado, corporeizado y encarnado dado que involucra a personas reales que viven en contextos sociales reales y complejos de los que no se pueden abstraer de manera significativa. A menos que lo hagamos desde el "reduccionismo ontológico", es decir, "the form in which objects of certain types can be shown to consist of nothing but objects of another type" (Searle, 1992, p. 113). De este modo, "if learning is nothing but a visible piece of observable behavior or information processing, all the intentional and contextual aspects of behavior vanish or are rejected under the claim that they are subjective ${ }^{2}$ or simply do not exist" (Sancho, 1995).

A partir de aquí, desde la perspectiva de la investigación post-cualitativa (St. Pierre, 2017) impulsada por los giros onto-ético-epistemológicos y metodológicos de los nuevos materialismos y los nuevos empirismos (St. Pierre, Jackson y Mazzei, 2016, entre otros), nos acercamos a algunas de las formas, modos, momentos y lugares de aprendizaje de docentes de infantil, primaria y secundaria que han participado en nuestra investigación, partiendo de sus cartografías de aprendizaje. Y lo hacemos no solo centrándonos en las interacciones con sus contextos, sino desde la propuesta de Barad (2007), para quien "la naturaleza es un devenir intra-activo (donde 'intra-acción' no es el clásico concepto reconfortante de 'interacción', sino que más bien implica la propia interrupción de la metafísica del individualismo que sostiene que hay objetos discretos con características inherentes)" (p. 422).. Esto nos permite sobrepasar la separación que tiende a realizar la investigación lógico-positivista entre el observador y lo observado, las personas y los objetos del mundo que las rodea, en este caso, los contextos de aprendizaje del profesorado, al considerar que están interconectados antes de que la mirada del investigador los separe (Barad, 2003).

El análisis de las intra-acciones de los espacios, tiempos, condiciones, expectativas, recursos, artefactos, etc., en los procesos del aprendizaje de docentes de distintos ciclos de enseñanza, representados de forma vi-

2 ¿Cómo podrían ser de otro modo si los que aprenden son sujetos, no objetos? 
sual, nos permite vislumbrar la constitución mutua de organismos enredados y los difuminados límites de los cuerpos y objetos, considerándolos fenómenos materiales discursivos. Para ello tomamos como referente la argumentación de Barad (1996) de que los cuerpos "humanos" no son intrínsecamente diferentes de los "no humanos".

Desde una mirada post-cualitativa, situamos este análisis desde la teoría y lo que ésta permite pensar para que, más que 'obtener resulta-

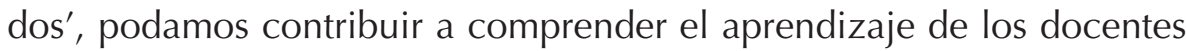
y sus implicaciones para la práctica y la formación inicial y permanente.

En enero/febrero de 2014, la revista Educational Researcher de la AERA (American Educational Research Association), dedicó una sección especial a lo que sus coordinadores Ilamaron "a continuing conversation as to what counts as quality research in education" (Southerland, Gadsden y Herrington, 2014, p. 7). La idea base partía de la importancia y necesidad de llevar a cabo una investigación educativa transparente y bien fundamentada, partiendo de la premisa "that highquality research should be fundamental to the improvement of educational policy and practice" (p. 7). Pero también señalaban que el sentido y la noción de 'transparencia', 'fundamentación' y 'calidad' están en relación con la perspectiva onto-ético epistemológica y metodológica en la que se sitúan los diferentes autores y líneas de pensamiento. De ahí que los distintos autores que contribuyeron a esta sección discutieran y fundamentaran sus visiones y convicciones (Wieman, 2014; Rudolph, 2014; Gutiérrez y Penuel, 2014), utilizando conceptos como "hard sciences", en general consideradas como 'las ciencias por excelencia' $y$ 'soft sciences or research in education', permanentemente en cuestión.

Desde la perspectiva de este artículo, el texto que nos resultó particularmente relevante fue el introductorio de Phillips (2014), en el que discute las características de la investigación en las "ciencias duras" y las "extremadamente duras en dominios 'blandos'". O como argumenta Berliner (2002), las "ciencias fáciles" y las "ciencias difíciles":

Easy-to-do science is what those in physics, chemistry, geology, and some other fields do. Hard-to-do science is what the social scientists do and, in particular, it is what we educational researchers do. In my estimation, we have the hardest to do science of them all! [...] We face particular problems and must deal with local conditions that limit generalizations and theory 
building - problems that are different from those faced by the easier-to-do sciences. (p. 18)

Pero, sobre todo, por la aproximación compleja y contextual mediante la cual Phillips aborda, en esta extensa cita, la problemática relacionada con el estudio del aprender.

Learning is a phenomenon that involves real people who live in real, complex social contexts from which they cannot be abstracted in any meaningful way. Difficult as it is for researchers to deal with (especially if they are suffering from physics envy), learners are contextualized. They do have a gender, a sexual orientation, a socioeconomic status, an ethnicity, a home culture; they have interests-and things that bore them; they have or have not consumed breakfast; and they live in neighborhoods with or without frequent gun violence or earthquakes, they are attracted by (or clash with) the personality of their teacher, and so on. It probably is the case, as some physical scientists have noted, that in a mature field of research, like physics, "it is obvious which variables are important and how to control them"; the problem is that in education, just about all the variables are relevant, and controlling them (even if possible let alone desirable) yields results that are difficult or impossible to generalize to the other almost infinite number of settings where these variables do, indeed, vary. I am not denying, of course, that physical scientists have to struggle to determine which variables to control, and to find out how actually to control them. But dealing with temperature, pressure, magnetic fields, and the like is one thing; dealing with culture, gender, socioeconomic status, human interests, and the like is quite another! This is why, while physics is a "hard science," education research is a very hard-an extremely hard-one. (p. 11)

Esta aproximación resulta fundamental dado que este artículo explora el aprender de los docentes, más que el aprendizaje docente. El aprender de un grupo de profesorado de educación infantil, primaria y secundaria a lo largo y lo ancho de la vida (Banks, Au, Ball, Bell et al., 2007). Es decir, no solo en la escuela, sino en los diferentes contextos, momentos, experiencias que componen su vida. Y nos lo planteamos desde una perspectiva post-cualitativa avanzada que se conecta con los giros ontoético-epistemológicos y metodológicos de los nuevos materialismos y los nuevos empirismos. 


\section{Marco teórico}

\section{Aproximación a los nuevos materialismos y los nuevos empirismos}

Para nosotros, la búsqueda de nuevas miradas onto-epistemológicas y metodológicas comenzó con la 'crisis de la ciencia positiva' de la década de 1960 (Barker, 1968; Gell-Mann, 1994; Maturana y Varela, 1980; Morin, 1982; 1990; Prigogine y Nicolis, 1987; Prigogine y Stengers; 1984; Von Bertalanffy, 1968, entre otros). Por su parte, St. Pierre, Jackson y Mazzei (2016) argumentan que desde el comienzo del siglo XXI ha aumentado el interés de los investigadores de ciencias sociales y humanidades por los denominados nuevos empirismos (Clough, 2009) y los nuevos materialismos (Coole y Frost, 2010; Dolphijn y van der Tuin, 2012). Una tendencia que plantea la crítica a los supuestos fundacionales y "las prácticas divisorias" (Foucault, 1982, p. 3) del pensamiento occidental que favorece oposiciones binarias como Igual/Otro, humano/ no-humano, mente/materia, cultura/naturaleza, conciencia/inconsciencia, trascendencia/inmanencia, idealismo/materialismo, observador/observado, investigador/investigado, etc.

En el empirismo clásico, que va de la mano del materialismo, se asume en general que lo dado, la materia (evidencia) "es una sustancia fija, bruta, inerte, y pasiva -objetos, cosas para ser utilizadas por los agentes humanos- quizás para ser observados o medidos en un estudio de ciencias sociales" (St. Pierre, Jackson y Mazzei, 2016, p. 99). Para estas autoras, la imbricación entre lo empírico y lo material lleva a la necesidad de repensar la ontología, la epistemología, la metodología y la ética en la que se basa la investigación. Ya que es la interconexión entre estas cuatro dimensiones la que pone de manifiesto que nuestra "forma de concebir el conocimiento y el ser es una cuestión profundamente ética, como lo es la relación entre lo humano y lo no humano" (Ibídem., 99). Pero sobre todo se plantean por qué y cómo ha sido posible y necesario pensar en estos nuevos marcos de pensamiento. En particular, señalan el imperativo ético de repensar la naturaleza del ser y la necesidad de la mayor curiosidad y experimentación que lo acompaña. Algo que resulta especialmente importante en un campo de extrema complejidad como es el de la educación y todos los fenómenos que la configuran.

En esta línea de pensamiento, y directamente relacionada con la perspectiva de este artículo, consideramos relevante el pensamiento de Ka- 
ren Barad, doctora en Física teórica y profesora de estudios feministas, filosofía e historia de la conciencia de la Universidad California, Santa Cruz. Barad (2003) considera la lucha del físico Neils Bohr contra los postulados meramente filosóficos, no basados en evidencias científicas, de Newton, Descartes y Demócrito. Para proporcionar una comprensión teórica de los hallazgos empíricos en el campo de la física atómica durante el primer cuarto del siglo XX, Bohr realizó la propuesta radical de que se requiere un marco epistemológico completamente nuevo. Aunque, según ella, "desafortunadamente, Bohr no explora las dimensiones ontológicas cruciales de sus percepciones, sino que más bien se centra en su importancia epistemológica" (Ibidem., 814). Por su parte, Barad propone la teoría del "realismo agencial", que define como "un relato de las prácticas tecnocientíficas y de otro tipo que toma en serio los estudios científicos feministas, antirracistas, postestructuralistas, queer, marxistas, estudios y conocimientos científicos, basándose específicamente en las contribuciones de Niels Bohr, Judith Butler, Michel Foucault, Donna Haraway, Vicki Kirby, Joseph Rouse, y otros" (Ibidem, 810-811).

Para Barad $(1996,2003)$ la realidad que nos rodea, que nos constituye y constituimos, no está configurada por objetos y sujetos como entes separados, sino por fenómenos. Los fenómenos se entienden como una maraña de seres vivos, objetos, espacios, tiempos y materias, que constantemente se reconfiguran, entretejen, relacionan y rearticular a partir de propias intra-acciones y las que se producen con otros fenómenos. Teniendo en cuenta que para Barad no solo son materia las cosas y los sujetos, sino también los discursos, lo que se piensa, lo que se imagina, lo posible y lo imposible.

Como hemos señalado, según Barad (2007) el universo engloba fenómenos que se caracterizan por la inseparabilidad ontológica de las agencias $^{3}$ intra-activas. Para Barad, la intra-acción se refiere a hechos causales no-arbitrarios y no-determinísticos a través de los cuales la materia-en-el-proceso-de-devenir es iterativamente envuelta en su continua materialización diferencial. Esta dinámica no viene marcada por un parámetro exterior llamado tiempo y tampoco tiene lugar en un contenedor llamado espacio, sino que las intra-acciones iterativas son dinámicas a través de las cuales temporalidad y espacialidad son producidas

3 Agency es una palabra inglesa de difícil traducción. Se refiere a la capacidad de ser, de actuar, en este caso, no solo de las personas sino de los seres vivos, los objetos e incluso las partículas que nos configuran a todos. 
e iterativamente reconfiguradas en la materialización del fenómeno y la (re)creación de los límites material-discursivos y sus exclusiones constituyentes. La noción de intra-acción representa un importante desafío a la metafísica individualista. Los fenómenos u objetos no preceden a su interacción, sino que emergen a través de intra-acciones particulares. Por lo tanto, los dispositivos (en términos de Foucault, 1991), que producen fenómenos, no son solo ensamblajes de humanos y no humanos, tal como postula la teoría del actor-red (Latour, 1996) sino que son la condición de posibilidad de lo 'humano' y lo 'no humano', no solo como conceptos ideacionales, sino en su materialidad. Los dispositivos son 'material-discursivo' en el sentido que producen determinados significados y seres materiales mientras que simultáneamente excluyen la producción de otros. Por lo tanto, lo que significa es que la materia es siempre material-discursivo.

La intra-acción sugiere que los límites que emergen en toda relación no son fijos, sino en continuo devenir. De este modo se descentra al humano para sugerir que somos siempre parte de algo y en continuo devenir con lo que nos rodea.

\section{Marco empírico}

El contenido de este artículo, al igual que el de algunos otros de este monográfico, presenta avances en la comprensión del aprender de los docentes, basados en el desarrollo del proyecto de I+D+I coordinado "APREN-DO - Cómo aprenden los docentes: implicaciones educativas y retos para afrontar el cambio social".

Desde la perspectiva post-cualitativa presentada en el apartado anterior, el foco de la investigación se centra en lo que nos permite pensar, en los conceptos que nos posibilita hacer emerger el proceso de realización de cartografías de aprendizaje elaboradas por un conjunto de docentes sobre sus tránsitos de aprender dentro y fuera de los centros de enseñanza.

Desde la investigación basada en las artes (Hernández, 2008), entendemos los métodos visuales (Banks, 2001; Dawani y Loots, 2015), en este caso las cartografías, como un 'artilugio de captura' que territorializa lo nuevo y lo singular y que, a modo de rizoma, pueden mostrar ensamblajes, multiplicidades o agregados de intensidades (Deleuze y 
Guattari, 2004). Al tiempo que los dibujos, imágenes o fotografías, aportados por los docentes para desarrollar sus cartografías, pueden ser utilizados como elementos desencadenantes y como formas de ayudar a vincular y poner de manifiesto conceptos abstractos (Mason, 2002; Sclater, 2003). Dado que lo que pretende esta investigación es tanto explorar las experiencias e intensidades que atraviesa el aprender de los docentes en activo participantes en la investigación (Coleman y Ringrose, 2014), como reinventar métodos de investigación para acercarnos a la fluidez, la multiplicidad y la vaguedad de las experiencias de aprendizaje (Law, 2004). Es decir, con este posicionamiento metodológico nos planteamos cómo indagar la multiplicidad de los mundos de aprendizaje del profesorado y las cuestiones planteadas por los propios métodos que no sólo 'capturan' estas realidades, sino que las construyen.

Han participado un total de 29 docentes de secundaria, 26 de ellos trabajan en tres centros y tres en distintos institutos. Todas las sesiones de investigación se realizaron de forma conjunta con cada grupo. En la primera sesión, para la realización de la cual se les había hecho llegar a los participantes un conjunto de documentos (referidos al sentido de la investigación, ejemplos de cartografías y el compromiso ético), los integrantes de cada grupo, acompañados por los investigadores, realizaron su cartografía. Al acabar se les invitó a que de forma individual narrasen el proceso seguido y las decisiones tomadas para hacerlo. Toda la sesión fue documentada audiovisualmente por miembros del equipo de investigación. A continuación, realizamos un análisis narrativo y dialógico de las cartografías, teniendo en cuenta toda la documentación textual, visual y audiovisual producida durante la sesión sobre cada participante. Creamos un documento para cada docente con su fotografía, la presentación que hizo sobre sí mismo, las imágenes del proceso de producción y de la cartografía, la transcripción de su relato y las notas de campo del investigador que acompañó el proceso creativo. A este documento añadimos un apartado titulado 'Lo que nos ha permitido pensar'. En la segunda reunión, les mostramos un vídeo editado por dos miembros del equipo destacando dos aspectos centrales: la relación entre el dentro y el fuera del centro y sus concepciones sobre aprendizaje. También compartimos nuestras resonancias con cada uno de los participantes, lo que nos llevó a continuar reflexionando sobre y a partir de sus cartografías.

En el caso de infantil y primaria, han colaborado 22 docentes de 12 centros educativos públicos y concertados. Primero celebramos un ta- 
Iler de creación cartográfica, para representar visualmente sus procesos de aprendizaje dentro y fuera de la escuela. Posteriormente, durante el curso el equipo de investigadores mantuvimos diferentes encuentros con las maestras y elaboramos un informe sobre cada una de las cartografías El informe incluía la fotografía de la cartografía, el texto reflexivo elaborado por cada autora, los diferentes momentos visuales de la creación y otro apartado sobre lo que nos había permitido pensar. Finalmente, organizamos otro taller, donde se expusieron todas las cartografías realizadas, una puesta en común del trabajo cartográfico de las maestras y de devolución de lo que había supuesto la experiencia para los investigadores.

\section{La re-construcción de la noción de aprender}

En coherencia con la mirada post-cualitativa adoptada en esta investigación explicitamos lo que nos ha permitido pensar el 'análisis' de algunas de las cartografías y de las conversaciones mantenidas sobre ellas con los docentes. Teniendo en cuenta que más que 'obtener resultados', nos proponemos contribuir a comprender el aprendizaje de los docentes y sus implicaciones para la práctica, la formación inicial y permanente. Y en coherencia con la perspectiva teórica dialogamos con el carácter contextual y complejo del proceso de aprender y en las intra-acciones de los docentes con el mundo que los rodea.

Uno de los objetivos de este artículo es vincular los nuevos materialismos y los nuevos empirismos con el aprender del profesorado. Es precisamente del aprender docente desde donde partimos para establecer los vínculos con las aportaciones teóricas, para comprender el alcance e implicación de las intra-acciones en el aprender docente. No es una tarea fácil. Primero por la falta de investigaciones que hayan abordado esta temática desde la perspectiva de los nuevos materialismos y empirismos. A lo que se suma la dificultad de manejar las intra-acciones en el contexto del aprender docente.

Ya nos ha advertido Biesta (2017) de las circunstancias que están contribuyendo a transformar la visión que tenemos sobre el aprender. De este modo, se trata de poner la atención en cómo lo humano y lo no humano intra-actúan en el aprender de los docentes, en la relación entre elementos independientes lo que, siguiendo a Jackson y Mazzei (2012), supone posicionamiento y performatividad, producción mutua de ac- 
ciones y como consecuencia un aumento del poder en las personas. Tal como hemos apuntado, la noción de intra-acción (Kerr, 2014) nos ofrece una forma completamente nueva de pensar las relaciones entre nosotros, con la materia, con los materiales, con la naturaleza y con los discursos. Cuando estas dimensiones diferentes se relacionan entre sí, nuestra capacidad para hacer cosas cambia, se transforma o emerge. Cuando dos cuerpos interactúan mantienen un nivel de independencia, cada entidad existe antes de que se encuentren entre sí. Pero cuando los cuerpos actúan de forma intraoperatoria, lo hacen de manera co-constitutiva. Los individuos se materializan a través de acciones internas y la capacidad de actuar emerge desde dentro de las relaciones y no fuera de ellas. Las interacciones desvían y difieren la responsabilidad, pero en las acciones internas, la responsabilidad se distribuye entre las entidades constitutivas. Aquí es donde entra en juego la noción de 'agencia', de capacidad de ser. La 'agencia' trata de la acción, la reconfiguración, el hacer y el ser. No preexiste por separado, sino que surge de las relaciones en las acciones internas. Pensar desde el concepto de intra-acción significa renunciar a las relaciones de causa y efecto, a la 'agencia' individual y a las dicotomías objeto-sujeto. Obtenemos así nuevos conocimientos de ética y justicia, ya que no son cosas predeterminadas, sino que siempre cambian y se desarrollan. Las intra-acciones cuestionan las fronteras y los límites rígidos y el tiempo lineal y, a su vez, nos ayuda a pensar en términos de simultaneidad. Derriba las paredes que contienen los pensamientos y acciones disciplinados para revelar límites artificiales que olvidamos que inventamos.

\section{Las intra-acciones en el aprender del profesorado de infantil, primaria y secundaria}

En relación con las cartografías de 5 docentes de Infantil y Primaria,la de Eneritz (Figura 1), maestra de música de educación primaria, es un ejemplo que incorpora elementos humanos (personas, colegas, amistades, alumnado) y no humanos: objetos, animales, espacios naturales, construcciones en la configuración de su aprender. Encontramos que la música constituye una de las intra-acciones emergentes en su cartografía. El elemento central que la ampara o protege al resto son unos cascos de música que, como el Traje de Sera cuya compra, según Jackson y Mazzei 
(2012), la hizo sentir poderosa, intra-actúa con los demás elementos en una producción mutua de acción, le dan poder, le dan capacidad de acción. Para ella la música es el elemento central que vertebra su seguridad, su aprender. Música que está presente en los momentos de relax, de tristeza y alegría. Con la música tiene una relación de continuidad y discontinuidad. Le da seguridad, la activa, la realiza. Junto a estos cascos aparecen otras fotografías que se relacionan con los grupos humanos donde ella vive y aprende. Una banda de música, un equipo de fútbol y que ella también hace extensible a su aprender docente, a la relación de colaboración que mantiene con sus colegas. También señala que uno de los espacios fundamentales es una casa que tiene en la naturaleza, donde celebra encuentros con amigos, con la familia. Un espacio que forma parte de su manera de vivir y que necesita para encontrar su equilibrio personal. Para finalizar destacar que, en otra fotografía, aparecen tres perros, que ella considera miembros de su grupo natural.

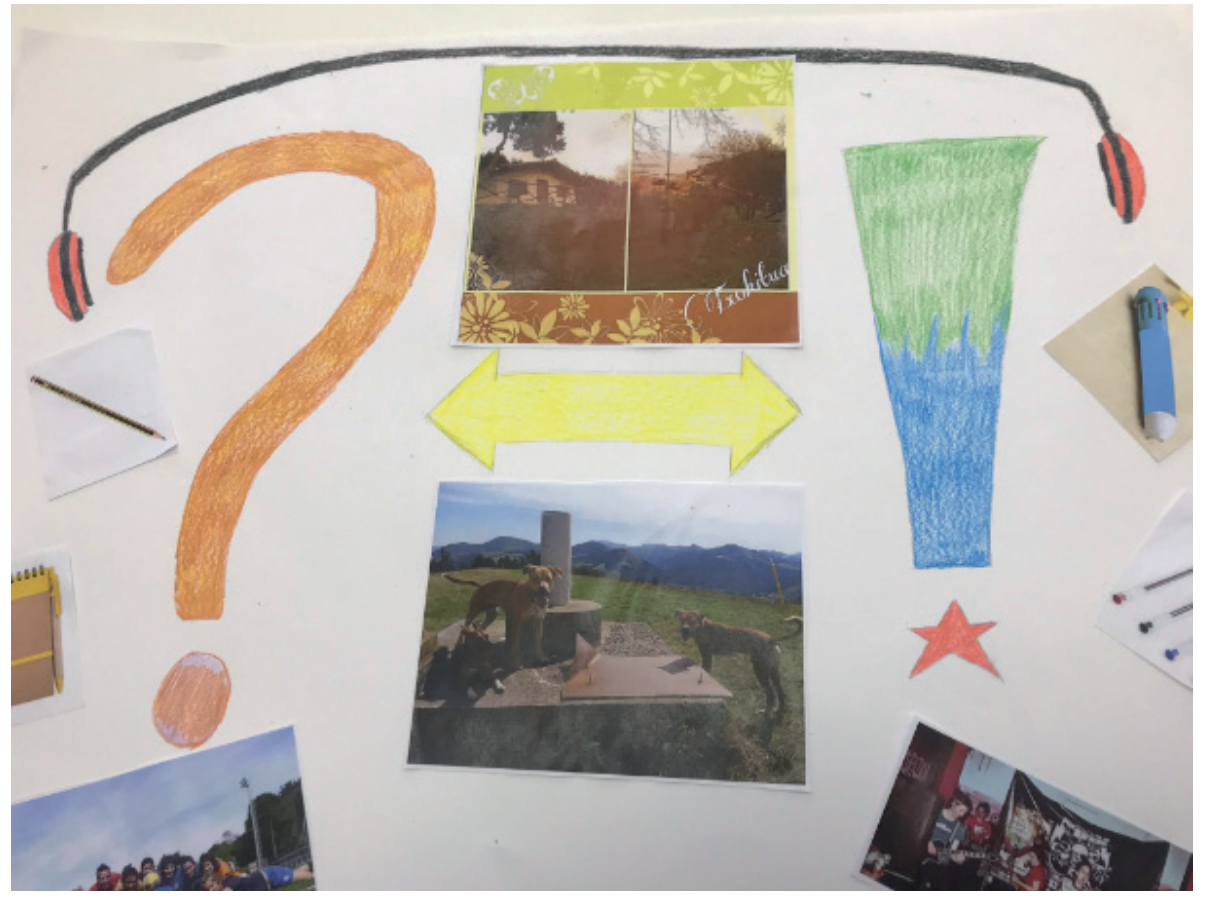

Figura 1. Cartografía de Eneritz. Fotografía de J.M. Correa

En la cartografía de Eneritz B (Figura 2), maestra que trabaja en educación infantil, encontramos la trikitixa, el instrumento musical, que ocupa 
el protagonismo central de la cartografía que le sirve para contarnos cómo ha sido un elemento fundamental en su aprender en los diferentes ámbitos de la vida. En su cartografía aparece como elemento principal unas notas musicales, donde ha incorporado las imágenes de su práctica docente. Para ella la trikitixa ha sido juego, le ha ayudado en su desarrollo personal superando vergüenzas, por ejemplo. Por otro lado, el haber sido concursante con este instrumento musical le ha proporcionado esfuerzo y disciplina y también respeto y amistad. Gracias a tocar la trikitixa ha conocido muchos lugares de Euskal Herria. Mirando a su cartografía y teniendo en cuenta al texto que escribió nos damos cuenta de que ha querido representar los sonidos diferentes de la trikitixa: en la parte izquierda (a la derecha del dibujo), con las notas bajas, con la armonía, ha querido representar la parte teórica. Con el lado derecho, con la melodía, los sentimientos. Para ella es necesario guardar equilibrio entre ambos, para comprender su proceso de aprendizaje. Con la trikitixa ha sentido, que si "alguno de los dos me falta me quedo medio desnuda".

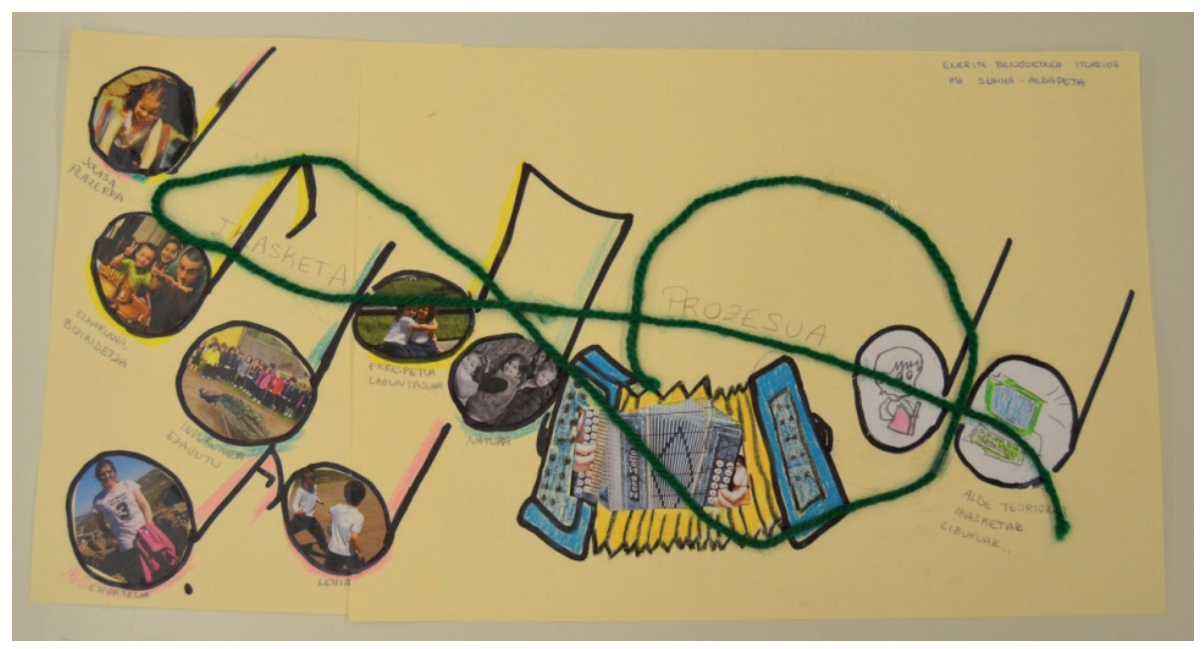

Figura 2. Cartografía de Eneritz B. Fotografía de J.M. Correa

Si encontramos en Eneritz los cascos de oír música y en Eneritz. B, la trikitixa, en el caso de Maider (figura 3), maestra de educación Primaria, lo que aparece es la pintura. Pintar un cuadro un lienzo, elementos independientes que preexisten y que crean algo nuevo, que aumentan su confianza y capacidad de acción, le produce bienestar y seguridad. En 
su cartografía aparece esta intra-acción en diferentes imágenes: dibujando en papel, pintando un grafiti y también tatuando.

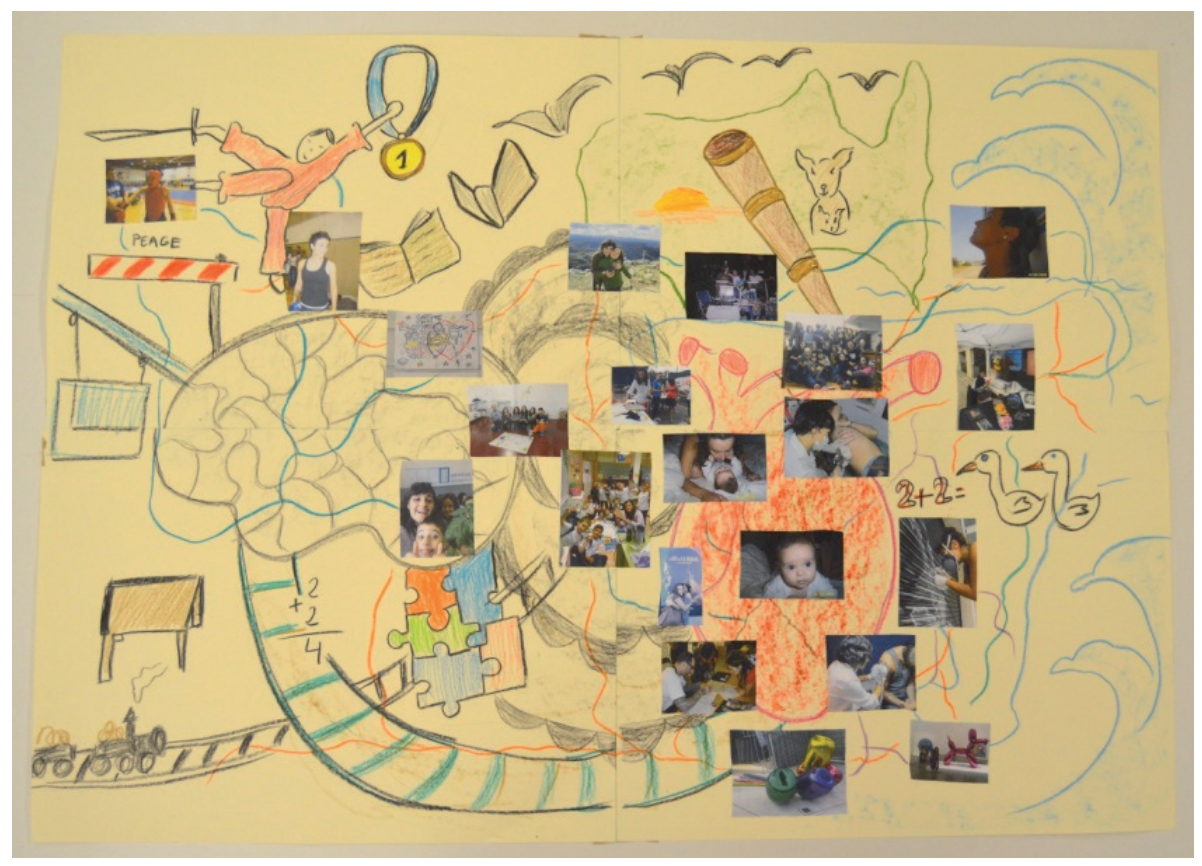

Figura 3. Cartografía de Maider. Fotografía de J.M. Correa

Imanol, un profesor con 14 años de experiencia, elaboró una cartografía (Figura 4) que tituló "Mis tripas", encontramos la mochila, que le acompaña en los viajes, cuando en verano parte a conocer otros rincones del mundo, otras culturas que estimulan y motivan sus procesos de aprender. En otra imagen de la parte delantera del cuerpo, salen de su boca las palabras "experiencias nuevas", "viajes", "cambios", "alumnos", "colegas"; y el cerebro ubicado en la zona del vientre y protegida con las manos como elemento motor de su persona. También hay fotografías sacadas por él mismo que representan diferentes contextos y relaciones. Y otra de su espalda donde aprece un texto escrito y el mapamundi donde se ven se ven viajes realizados por tres continentes. Imanol, por ejemplo, se refería así a los viajes: "Cada vez que viajo siento que crezco y para mi crecer es sinónimo de aprender, mejorar, cambiar... Cuando te pones en relación con otra cultura que es otra realidad que no es la tuya, aprendes y aprendes mucho. Apren- 
Intra-acciones en el aprender de docentes de infantil, primaria y secundaria juana María Sancho-Gil y José Miguel Correa-Gorospe

des a observar, aprender a escuchar, aprendes a valorar... En definitiva, aprendes a conocerte.

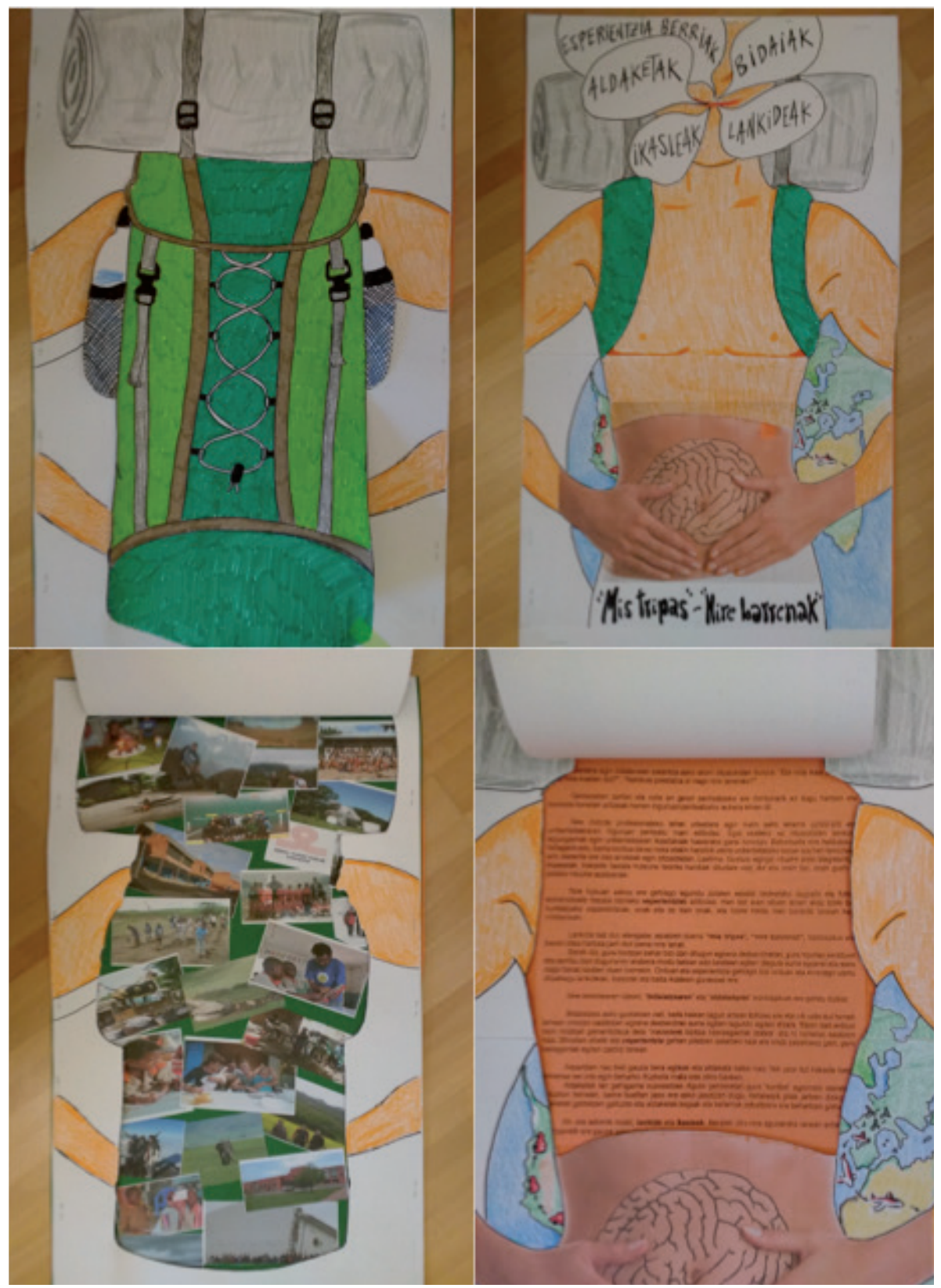

Figura 4. Cartografía de Imanol. Fotografía de J.M. Correa. 
En el caso de Daniel (Figura 5) en su cartografía encontramos una foto de un mapa del País Vasco con un lema que dice: "cada escuela es un viaje acompañado". Hace referencia a la experiencia del viaje al trabajo, que durante tantos años ha tenido que realizar para desplazarse hasta las diferentes escuelas donde ha estado trabajando. Destaca en este aprendizaje un elemento material: el coche, que para él tiene un valor especial, un espacio donde ha podido intercambiar experiencias, desarrollar un proceso relacional con otros compañeros con quienes se desplazaba a los centros de trabajo. Cuando viajan, una hora de ida y otra de vuelta, con otros colegas, tienen oportunidad de revisar lo que ha ocurrido en las aulas, el acierto o no de las actividades que cada día van desarrollando con escolares. Es compartir coche con parte de la plantilla docente, gente afín, donde aprendes a abrirse, a criticar, a ser criticado también. Sabe que le están criticando, porque no gustan sus formas de hacer las cosas. Revisa sus actividades escolares, la relación con familia y alumnado, recibe aportaciones e intercambiar ideas con quienes le acompañan en el viaje.

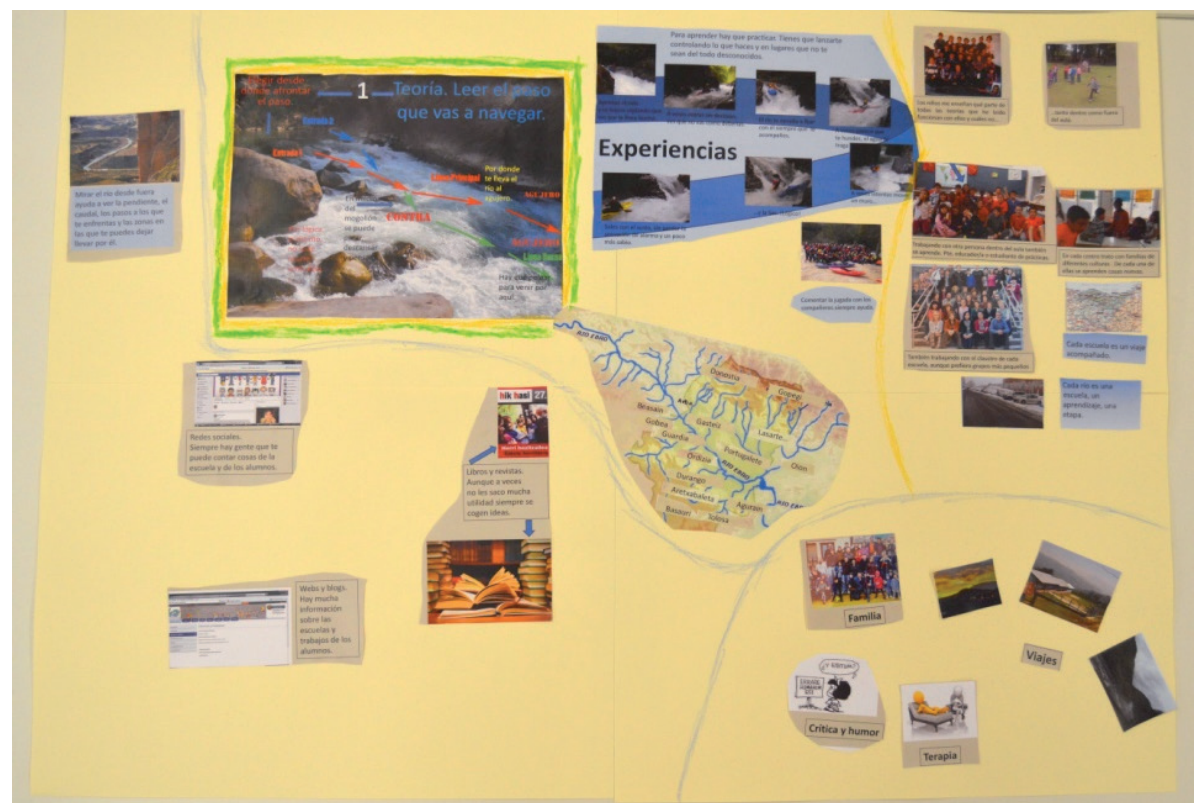

Figura 5. Cartografía de Daniel. Fotografía de J.M. Correa.

Por lo que se refiere a los docentes de secundaria, el diálogo sobre lo que sus aportaciones nos han permitido pensar, se ha centrado en 
9 cartografías llevadas a cabo por otros tantos docentes (6 mujeres y 3 hombres), pertenecientes a los 3 centros que han colaborado en la investigación.

La evidencia de que el aprendizaje es un fenómeno que tiene que ver con las personas como un todo, no solo con sus cerebros, que viven en contextos sociales complejos y reales, de los que no se pueden abstraer de manera significativa (Phillips, 2014, p. 11) queda claramente manifiesta en todas las personas que han participado en esta investigación. Todas ellas identifican contextos sociales significativos (familia, educación formal, práctica profesional...), pero lo que varía es el sentido que tienen para ellas y el sentido y el papel que les otorgan. También queda de manifiesto que, como hemos argumentado anteriormente, sus realidades de aprendizaje están configurados por una maraña de seres vivos, objetos, espacios, tiempos, materia y discursos y lo que se piensa, se imagina, lo posible y lo imposible. Todo ello constantemente reconfigurado, entretejido, relacionado y rearticulado a partir de las propias intra-acciones y de las que se producen con otros fenómenos.

Hay que tener en cuenta que, aunque la cartografía se presente como un producto final, y por ello sus autores se esfuerzan por darle una forma estética y entendible para el otro, su proceso de elaboración -como sucede en cualquier texto, puede mostrar procesos matéricos profundamente enmarañados. Este es el caso de Rodolfo, profesor de educación visual y plástica del INS MC. Como podemos ver en la figura 7, en su cartografía final sitúa sus aprendizajes en espacios claramente definidos e interconectados, a través del espacio central de la creación, en los que se siente académica, artística y profesionalmente legitimado.

Sin embargo, el proceso de producción de su cartografía, parte de una experiencia corporeizada, familiar, cultural, histórica y material. En el relato que acompaña su cartografía Rodolfo manifiesta que "está basada en un espacio físico, que existe. Es un espacio completo físico, muy caótico, que hay en mi pueblo, donde nací. Es un espacio subterráneo, que para mí se ha convertido en un espacio de reflexión y al mismo tiempo este espacio caótico me sirve a mí para recolocarme en ciertos aspectos mentales que tengo o cosas que me motivan, que me impresionan, que me cautivan en mi proceso artístico o como profesor, entonces lo he utilizado para hacer esta cartografía" (Figura 6). 


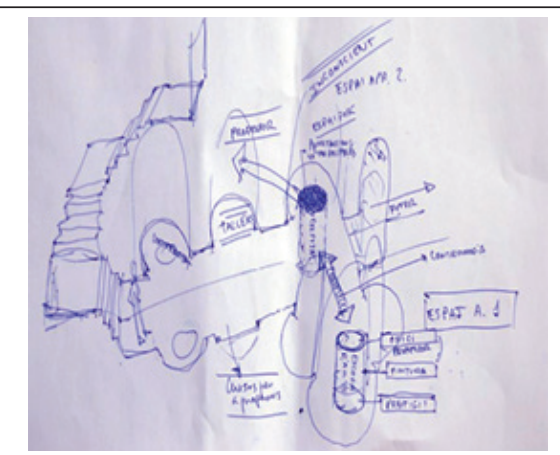

Figura 6. Proceso de producción de la cartografía de Rodolfo INS MC. Fotografía de J. M. Sancho.

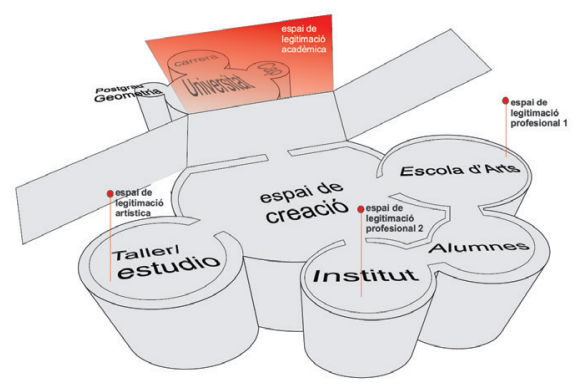

Figura 7. Cartografía de Rodolfo INS MC. Fotografía de J. M. Sancho.

En las visualizaciones cartográficas sobre su aprender, el profesorado de secundaria transmite la idea de movimiento, fluidez, intra-acciones, crecimiento, iteración, ... Ya sea a través de un conjunto de olas en movimiento sin fin (Rosalía INS MVM) -figura 8, del crecimiento paulatino de un árbol, centrado en una hoja (Silvia INS MVM) -figura 9, del constante fluir del río (Maribel INS T)- figura 10, de fuerzas expansivas de distintas intensidades (Sara INS MC)- figura 11, de un camino que se expande y que marca los límites entre lo sabido y lo desconocido (Josep, INS MVM) -Josep 12, las espirales continuas (Rocío INS MC y Silvia INS T)- figuras 13 y 14 . Incluso en las visualizaciones aparentemente más estáticas, como en el campo de fútbol (Lluís INST T) -figura 15 y en y entre los espacios de formación, creación y profesión (Rodolfo INS MC) figura 7 se producen movimientos e intra-acciones $\sin$ fin.

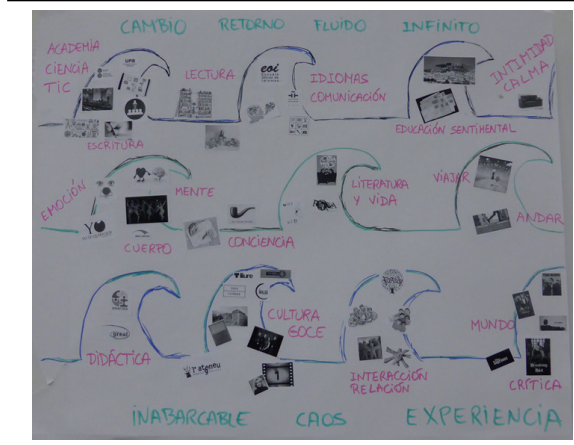

Figura 8 . Cartografía de Rosalía INS MVM. Fotografía de J.M. Sancho

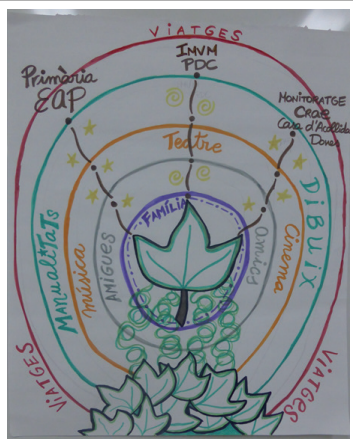

Figura 9. Cartografía de Sílvia INS MVM. Fotografía de J.M. Sancho 


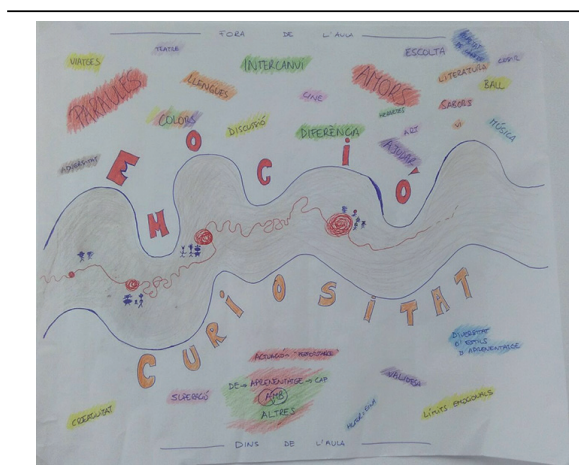

Figura 10. Cartografía de Maribel INS

T. Fotografía de J.M. Sancho
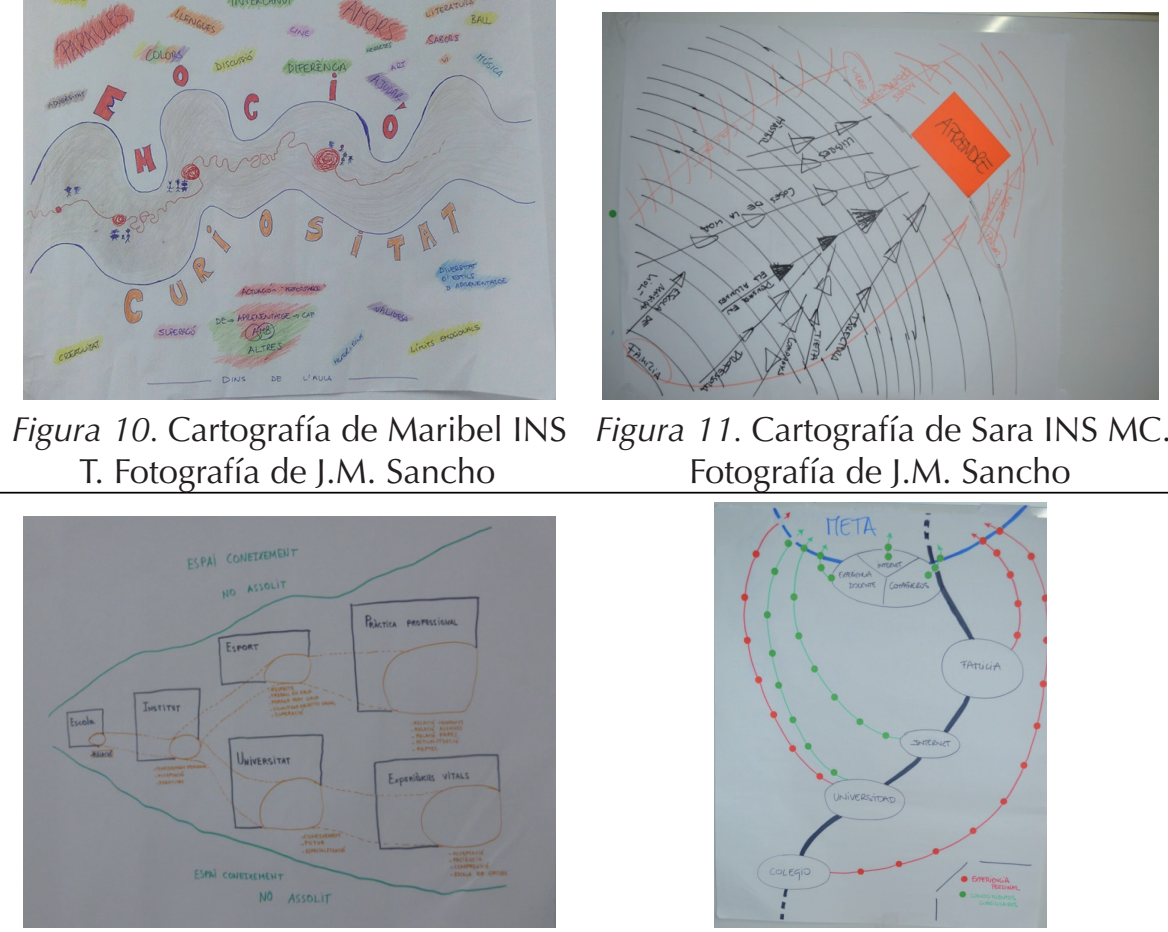

Figura 11. Cartografía de Sara INS MC. Fotografía de J.M. Sancho

Figura 12. Cartografía de Josep INS MVM. Fotografía de J.M. Sancho

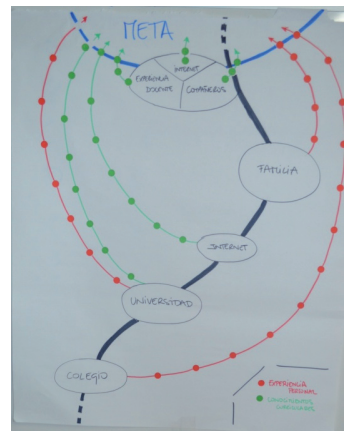

Figura 13. Cartografía de Rocío INS

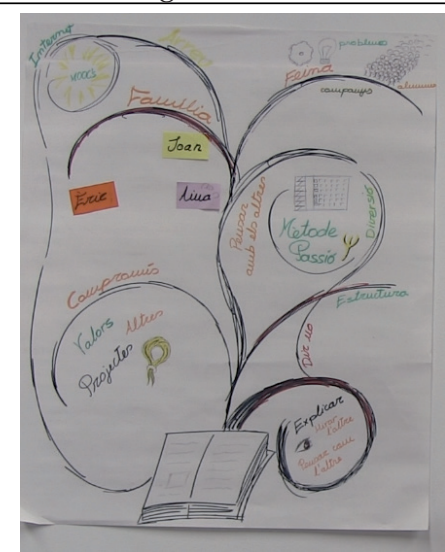
MC. Fotografía de J.M. Sancho

Figura 14. Cartografía de Sílvia INS T. Figura 15. Cartografía de Lluís INS T. Fotografía de J.M. Sancho

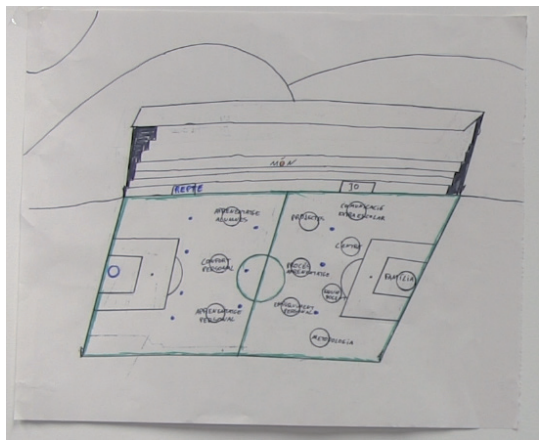
Fotografía de J.M. Sancho

Las personas que han colaborado en esta investigación no establecen límites ni barreras entre los diferentes momentos, etapas, entornos, 
situaciones, experiencias por los que transitan y han transitado a lo largo de la vida. Y reconocen aprender en todas ellos. "Pongo anécdotas de mi propia vida, mi experiencia en el colegio, de cómo me enseñaron a mí, anécdotas universitarias, de mí propia familia" (Rocío INS MC). Rosalía (INS MVM), profesora de lengua castellana, vincula su iniciativa de intercambio de cartas y visitas entre estudiantes de la ESO de distintos institutos a su experiencia adolescente de veranos en el pueblo de su familias "el invierno extrañando a esos amigos y lo que hacíamos era escribir cartas larguísimas y por eso he puesto las cartas y yo creo que lo que yo sentía cuando me llegaba una carta, de esa emoción viene el proyecto el cual yo monté para los alumnos de primero de ESO".

Tal como argumenta Barad (1996) sus aprendizajes se constituyen como fenómenos materiales discursivos hechos de organismos, objetos, tecnologías, tiempos, espacios, situaciones, experiencias, valores y conceptos enredados. Como expresa Sílvia (INS T) "yo aprendo siempre", porque considera que aprender es como respirar.

En esta maraña, en la que se genera un importante volumen de aprendizajes subliminales (Mlodinow, 2013), que pueden facilitar o inhibir el aprendizaje profesional y la capacidad de transformar y transformarse, la familia ocupa un lugar fundamental. Para Sara, la familia "lo engloba todo". Lluís (INS T), que la considera como "lo más importante", la sitúa en la portería, en el lugar para 'parar los goles'. Mientras para Sílvia (INS MVM), que la sitúa en el centro de la hoja que forma parte del crecimiento del árbol que para ella es el aprender, representa su nutriente principal. Esto nos llevó a reflexionar sobre el papel fundamental de la familia en el proceso de aprender y en la desigualdad de condiciones con la que el alumnado llega ya a la educación infantil, y no digamos a la secundaria.

Los espacios de formación, tanto inicial como permanente, han sido señalados y visualizados de formas distintas por cada docente. Pero sobre todo resignifican la importancia del aprendizaje de y en la práctica, tanto con sus colegas como con sus estudiantes. "He puesto mis compañeros de trabajos, mi familia, mis alumnos, porque a veces si tú escuchas lo que dicen, te dicen cosas que no se te habían ocurrido a ti antes, pero nunca, que te abren muchos caminos, de cómo enfocar las cosas, en las que no habías pensado precisamente, por eso es importante escuchar al otro, en la interacción, dejar hablar" (Rosalía INS MVM). 
Fuera de los espacios institucionales, formales e informales, señalan, visualizan, lo que Maribel denomina "los amores, en un sentido amplio: mis amigos, mis compañeros, la gente que me rodea y de la cual me nutro y a la que intento nutrir también". Ese conjunto de personas que pueblan nuestras vidas y que nos pueden dejar, y en las que podemos dejar, distintos tipos de huellas. A ello hay que añadir todos los recursos tecnológicos que pueblan y constituyen nuestro mundo. Los libros, como "literatura y vida" (Rosalía INS MVM). Como símbolo del saber y base del aprendizaje (Sílvia INS T) y en los últimos años Internet, a quien consideran una fuente "muy valiosa de aprendizaje" (Rocío INS MC), "mi entorno de aprendizaje más importante" (Sílvia INS T), "porque me llega mucha información y también aprendo cosas y las incorporo a mis clases" (Rosalía INS MVM). En la segunda sesión en el INS MC, esta constatación llevó a una de las profesoras a reflexionar sobre la aparente incoherencia que representaba para ella, 'descubrir' que ahora estaba aprendiendo de otra forma, pero seguía esperando que los estudiantes aprendieran, y ella enseñase, como a ella le habían enseñado.

Finalmente, en las cartografías aparecen una serie de los aspectos enmarañados e interconexos que revisten un gran interés y resultan menos explorados en el campo del aprendizaje: 1) El conjunto de experiencias y actividades culturales señaladas y visualizadas por el conjunto de docentes: viajes, cine, teatro, baile, música, deportes, aficiones, etc. 2) El cúmulo de valores y conceptos: reto, método y pasión, compromiso, valores, proyecto, curiosidad, emoción, colores, superación, creatividad, diferencia, ayuda, bienestar, cuerpo, conciencia, andar, cultura goce, educación sentimental, vivir, pensar. Aspectos que, sin embargo, pueden marcar la diferencia al sentido profundamente contextual, biográfico y corpóreo del aprendizaje.

\section{A modo de conclusión: Implicaciones y desafíos}

Las perspectivas teóricas de los nuevos materialismos y los nuevos empirismos representan una nueva onto-ético-epistemología que ayudan a visibilizar la complejidad de las relaciones educativas, del aprender de los docentes y sus implicaciones éticas.

Los nuevos materialismos tienen un efecto perturbador para quienes 
investigan y para la propia investigación educativa, al quebrar las representaciones tradicionales de las relaciones con el conocimiento, no solo por considerar la capacidad de acción de la materia, sino también por lo que supone la propia pérdida de la centralidad de lo humano, tal como hasta ahora lo habíamos considerado.

También conlleva una profunda crisis en la representación y validación del conocimiento en la medida que no se busca la descripción de la naturaleza, sino el sentido de la participación de la propia materia, el análisis de su capacidad de acción y la necesidad de poner el foco en su rol activo. Pasando a considerar lo humano y no humano como elementos que se constituyen mutuamente.

Situar esta investigación en esta perspectiva nos ha enfrentado a un gran reto. Pero nos ha posibilitado evidenciar, junto con el colectivo de docentes, la contextualidad, corporeidad, fluidez y devenir del aprendizaje. Un proceso que no solo tiene lugar en los centros y las aulas, sino que se configura como un complejo entramado en continua intra-acción con todo lo que nos rodea.

Esta constatación empírica, como apuntan los propios colaboradores, conlleva la necesidad de repensar -en profundad y extensión, por una parte, los modelos de formación inicial y permanente del profesorado, en general, basados en exceso en la 'transmisión' de conocimiento disciplinar y prescriptivo. Y por la otra, los discursos y las prácticas de los centros de enseñanza que tienden a seguir considerando el aprendizaje como una abstracción y a separar a los aprendices de sus contextos sociales, materiales y biográficos.

Finalmente, también nos lleva a la importancia de plantearnos preguntas diferentes sobre el aprender y a poner en práctica modos de indagación más corporeizados y performativos, que nos acerquen a la complejidad de los fenómenos de la educación.

\section{Referencias}

Banks, J. A., Au, K. H., Ball, A. F., Bell, P., Gordon, E. W., Gutiérrez, K. y Zhou, M. (2007). Learning in and out of school in diverse environments. life-long, life-wide, life-deep. The LIFE Center for Multicultural Education. Recuperado de https://www.google.com/ search?q=Learning+in+and+out+of+school+in+diverse+environments.+life-long\%2 C+life-wide\%2C+life-deep\&ie=utf-8\&oe=utf-8\&client=firefox-

Banks, M. (2001). Visual Methods in Social Research. London: SAGE Publications. 
Barad, K. (1996). Meeting the Universe Halfway: Realism and Social Constructivism without Contradiction. En L. H. Nelson y J. Nelson (Eds.), Feminism, science, and the philosophy of science (pp. 161-194). Dordrecht: Springer.

Barad, K. (2003). Posthumanist Performativity: Toward an Understanding of How Matter Comes to Matter. Signs: Journal of Women in Culture and Society, 28(3), 801-831.

Barad, K. (2007). Meeting the universe halfway: Quantum physics and the entanglement of matter and meaning. Durham, NC: Duke University Press.

Barker, R. G. (1968). Ecological Psychology: Concepts and methods for studying the environment of human behavior. Palo Alto, CA.: Stanford University Press.

Berliner, D. C. (2002). Comment: Educational Research:The Hardest Science of All. Educational Researcher, 31(8), 18-20. doi:10.3102/0013189x031008018

Biesta, G. (2016). Democracia, ciudadanía y educación: de la socialización a la subjetivación. Foro de Educación, 14(20), 21-34. doi: http://dx.doi.org/10.14516/ fde.2016.014.020.003

Clough, P. T. (2009). The new empiricism: Affect and sociological method. European Journal of Social Theory, 12, 43-61. doi:10.1177/1368431008099643

Coleman, R., y Ringrose, J. (Eds.) (2014). Deleuze and Reseach Metodologies. Edinburgh: Edinburgh University Press.

Coole, D., y Frost, S. (2010). Introducing the new materialism. En D. Coole y S. Frost (Eds.), New materialisms: Ontology, agency, and politics (pp. 1-43). Durham, NC: Duke University Press.

Dawani, S. y Loots, G. (2015). Doing research together: creating spaces of collaboration with young people using visual methods. Visual Methodologies. A postdisciplinary journal, 3 (1), 1 -17. doi: https://doi.org/10.7331/ vm.v3i1.44

Deleuze, G., y Guattari, F. (2004). Mil mesetas . Capitalismo y esquizofrenia. Valencia: Pre-Textos.

Dolphijn, R., y Van der Tuin, I. (2012). New materialisms: Interviews and cartographies. Ann Arbor, MI: Open Humanities Press.

Foucault, M. (1988). El sujeto y el poder. Revista Mexicana de Sociología, 50(3), 3-20.

Foucault, M. (1991). El juego de Michel Foucault. En M. Foucault, Saber y verdad (pp. 127-162). Madrid: Ediciones La Piqueta.

Gell-Mann, M. (1994). El Quark y el Jaguar. Aventuras en lo simple y lo complejo. Barcelona: Tusquets.

Gutiérrez, K. D., y Penuel, W. R. (2014). Relevance to Practice as a Criterion for Rigor. Educational Researcher, 43(1), 19-23. doi:10.3102/0013189x13520289

Hernández, F. (2008). La investigación basada en las artes. Propuestas para repensar la investigación en educación. Educatio Siglo XXI, 226, 85-118.

Jackson, A.Y., y Mazzei, L. A. (2012). Thinking with theory in Qualitative Research. London: Routledge.

Kerr, S. (2014/11/14). Three Minute Theory: What is Intra-Action?[Archivo de video] Recuperado de https://www.youtube.com/watch? $v=v 0 S n s t) o E e c$

Latour, B. (1996). On actor-network theory. A few clarifications plus more than a few 
Intra-acciones en el aprender de docentes de infantil, primaria y secundaria

complications. Soziale Welt, 40, 369-381. Recuperado de http://www.bruno-latour. fr/sites/default/files/P- 67\%20ACTOR-NETWORK.pdf

Law, J. (2004). After Method: Mess in Social Science Research. London y New York: Routledge.

Mason, J. (2002). Qualitative interviewing: asking, listening and interpreting. En T. May (Ed.), Qualitative research in action (pp. 225-242). London: Sage.

Maturana, H., y Varela, F. (1980). Autopoiesis and cognition: The realization of the living. Dordrecht, Holland: D. Reidel Publishing Company.

Mlodinow, L. (2013). Subliminal. Cómo tu inconsciente gobierna tu comportamiento. Barcelona: Crítica.

Morin, E. (1982). Science avec conscience. Paris: Fayard.

Morin, E. (2001). Introducción al Pensamiento Complejo. Barcelona: Gedisa.

Phillips, D. C. (2014). Research in the Hard Sciences, and in Very Hard "Softer" Domains. Educational Researcher, 43(1), 9-11. DOI: 10.3102/0013189X13520293

Prigogine, I., y Nicolis, G. (1997). La estructura de lo complejo. Madrid: Alianza.

Prigogine, I., y Stengers, I. (1984). Order out of Chaos: Man's new dialogue with nature. Toronto. New York, N.Y.: Bantam Books.

Rudolph, J. L. (2014). Why Understanding Science Matters: The IES Research Guidelines as a Case in Point. Educational Researcher, 43(1), 15-18. doi:10.3102/0013189x13520292

Sancho, J. M. (1995). Looking for the 'Right' Answers or Raising the 'Right' Questions? A Dialogical Approach to Automating Instructional Design. En R. D. Tennysony A. E. Barron (Ed.), Automating Instructional Design: Computer-Based Development and Delivery Tools (pp. 79-99). Springer-Verlag. NATO ASI Series F: Computer and Systems Sciences, Vol. 140.

Sclater, D. (2003). The arts and narrative research. Qualitative Inquiry, 9(4), 621-625.

Searle, J. R. (1992). The Ridescovery of the Mind. Cambridge, MA.: The MIT Press.

Southerland, S.A., Gadsden, V. L., y Herrington, C. D. (2014). Editors' Introduction: What Should Count as Quality Education Research? Continuing the Discussion. Educational Researcher, 43(1), 7-8. doi:10.3102/0013189x13519962

St. Pierre, E. A. (2017). Haecceity: Laying Out a Plane for Post Qualitative Inquiry. Qualitative Inquiry, 23(9), 86- 698. doi: 10.1177/1077800417727764

St. Pierre, E. A., Jackson, A. Y. y Mazzei, L. A. (2016). New Empiricisms and New Materialisms. Conditions for New Inquiry. Cultural Studies $\leftrightarrow$ Critical Methodologies, 16(2) 99-110.

Von Bertalanffy, L. (1968). General system theory; foundations, development, applications. New York: G. Braziller.

Wieman, C. E. (2014). The Similarities between Research in Education and Research in the Hard Sciences. Educational Researcher, 43(1), 12-14. doi:10.3102/0013189x13520294 


\section{Financiación}

Investigación parcialmente financiada por Ministerio de Economía y Competitividad / EDU2015-70912-C2-1-R; EDU2015-70912-C2-2-R.

\section{Agradecimientos}

Esbrina - Subjetividades, visualidades y entornos educativos contemporáneos (2017SGR 1248).

Elkarrikertuz — Innovación, tecnologías digitales y transformaciones culturales y educativas. (IT887-16)

REUNI+D -Red Universitaria de Investigación e Innovación Educativa. Cambios Sociales y Retos para la Educación en la Era Digital (EDU201568718-REDT). 
